\title{
MgrA regulates interaction of Staphylococcus aureus with mucin
}

\author{
by
}

Connor P. Parker ${ }^{1}$, Nour Akil ${ }^{1}$, Cullen R. Shanrock ${ }^{1}$, Patrick D. Allen ${ }^{1}$, Anna L. Chaly ${ }^{1}$, Heidi A. Crosby ${ }^{2}$, Jakub Kwiecinski ${ }^{2}$, Alexander R. Horswill ${ }^{2}$, and Anthony J. Fischer ${ }^{1}$

Title: 62 characters with spaces

Abstract word count: 249, Max 250

Word count: 3456

References: 42

21

Keywords: Staphylococcus aureus, Mucin, Submucosal Gland, MgrA, Adhesin

Running Title: MgrA regulates mucin interaction

\section{Author Affiliations}

1. Stead Family Department of Pediatrics, University of lowa Carver College of Medicine, lowa City, IA

2. Department of Immunology \& Microbiology, University of Colorado Anschutz Medical Campus, Aurora, CO

\section{Correspondence}

Anthony J. Fischer

Stead Family Department of Pediatrics

6314 PBDB

University of lowa Carver College of Medicine

lowa City, IA 52242

Email: anthony-fischer@uiowa.edu

Fax: 319-356-7776

Phone: 319-356-6992 


\section{Abstract}

45 Background: To defend the lungs, mucus adheres to bacterial cells and facilitates their

46 removal by ciliary transport. Our goals were to measure the affinity of mucus for the respiratory

47 pathogen Staphylococcus aureus and identify bacterial genes that regulate this interaction.

48 Methods: $S$. aureus was added to pig tracheas to determine whether it binds mucus or

49 epithelial cells. To quantify its affinity for mucus, we developed a competition assay in microtiter 50 plates. Mucin was added over a dose range as an inhibitor of bacterial attachment. We then

51 examined how transcriptional regulator MgrA and cell wall transpeptidase sortase (SrtA) affect

52 bacterial interaction with mucin.

53 Results: In pig tracheas, $S$. aureus bound mucus strands from submucosal glands more than

54 epithelial cells. In microtiter plate assays, $\Delta$ stt $A$ failed to attach even in the absence of mucin.

55 Mucin blocked wild type S. aureus attachment in a dose-dependent manner. Higher

56 concentrations were needed to inhibit binding of $\Delta m g r A$. Co-deletion of ebh and sraP, which

57 encode surface proteins repressed by MgrA, suppressed the $\Delta m g r A$ binding phenotype. No

58 differences between $\Delta m g r A$ and wild type were observed when methylcellulose or heparin

59 sulfate were substituted for mucin, indicating specificity.

60 Conclusions: Mucin decreases attachment of S. aureus to plastic, consistent with its

61 physiologic role in host defense. S. aureus deficient in MgrA has decreased affinity for mucin.

62 Ebh and SraP, which are normally repressed by MgrA, may function as inhibitors of attachment

63 to mucin. These data show that specific bacterial factors may regulate the interaction of $S$.

64 aureus with mucus.

65 


\section{Introduction}

68 Mucus is an important defense against respiratory infections. It is a gel-like material comprised

69 of hydrated mucin proteins and inorganic salts produced on epithelial surfaces. In large animals,

70 airway mucus is produced in the form of strands arising from submucosal glands (1-3). Inhaled

71 particles and pathogens can become entrapped in mucus. After the release of mucus strands

72 from submucosal gland ducts or goblet cells, ciliated cells transport mucus together with any

73 particles trapped in the mucus, expelling them from the airway (1). Although mucus is

74 colloquially described as "sticky," its adhesive properties are seldom quantified. Proper

75 physiologic function of mucus would require that it have some affinity for a diverse repertoire of

76 particles and pathogens, but not excessively adhere to the epithelium or gland duct surfaces. In

77 cystic fibrosis (CF), mucus may interact with pathogens, but it fails detach from gland ducts and

78 is unable to be carried away by ciliary beating. This results in impaired transport of mucus out of

79 the airway (3). In CF pigs, mucus strands fail to detach from airway submucosal glands and

80 becomes pathologically immobile. Thus, it has been hypothesized that mucus stasis may

81 contribute to the establishment of the chronic bacterial infections in CF.

83 S. aureus is a typical pathogen in CF, as approximately $70 \%$ of people with CF are infected with

84 the bacteria $(4,5)$. Among CF pathogens, $S$. aureus has the highest infection incidence in the

85 preschool age group, making it one of the earliest organisms in CF (4). Furthermore, patients

86 infected with methicillin-resistant $S$. aureus (MRSA) experience faster CF disease progression

87 (6-8). Currently there is no vaccine against $S$. aureus, and eradication of incident infections

88 (especially MRSA) remains challenging (9). Hence, there is a critical need to understand how $S$.

89 aureus initially infects the CF airway. 
93 There is some prior evidence the $S$. aureus associates with mucus. Mucins from saliva,

94 nasopharynx, and conjunctiva are known to bind $S$. aureus (10). In animals infected intranasally

95 with $S$. aureus, bacteria bind to mucus from anterior and posterior turbinates without direct

96 binding to the underlying epithelium (11). However, the molecular basis for $S$. aureus

97 attachment to mucin is not fully understood. S. aureus expresses a variety of surface proteins,

98 collectively known as adhesins, that are implicated in the pathogenesis of other serious

99 infections like endocarditis and sepsis. We hypothesized that bacterial genetic factors regulating

100 adhesins control S. aureus attachment to mucus. In this study, we observed whether S. aureus

101 attaches to mucus in pig tracheas and we developed a method to quantify the affinity of $S$.

102 aureus for mucin by a competition assay. We used this approach to measure the interaction of

103 mucin with S. aureus strains with suspected defects in attachment. We examined two candidate

104 regulators of bacterial attachment: Sortase A (SrtA), a transpeptidase enzyme required for

105 surface display of proteins (12), and multiple gene regulator A (MgrA), a transcriptional regulator

106 of surface adhesins, including the giant staphylococcal surface proteins Ebh and SraP (13-15). 


\section{Materials and methods}

\section{Bacteria strains and growth conditions}

111 Bacterial strains and plasmids used are listed in the Table 1. S. aureus was grown overnight in 112 tryptic soy broth (TSB), supplemented with $10 \mu \mathrm{g} / \mathrm{ml}$ of chloramphenicol (Cam) to maintain the 113 sGFP-expressing plasmid pCM29. For construction of S. aureus mutants, tryptic soy agar with

114 Cam or with tetracycline (Tet, $1 \mu \mathrm{g} / \mathrm{ml}$ ) were also used. To construct mutant strains,

115 bacteriophage transduction between $S$. aureus strains was performed with phage $80 \alpha$ or 11 as

116 described previously (16). To generate the sortase mutant $\mathrm{AH} 4480$, the $\Delta s r t A$ mutation

117 containing a Tet $^{R}$ cassette was transduced from the previously described $\Delta s r t A$ mutant in the $S$.

118 aureus strain RN4220.

Trachea preparations. Animal studies were approved by the University of lowa Animal Care

121 and Use Committee. Male and female newborn pigs were obtained from Exemplar Genetics.

122 The pigs were sedated with ketamine and acepromazine or xylazine and euthanized with I.V.

123 Euthasol. We removed $1 \mathrm{~cm}$ tracheal segments following euthanasia, made a longitudinal cut

124 on the ventral surface, and pinned the trachea flat to expose the mucosal surface. To mimic the

125 CF electrolyte transport defect, we used conditions that impair chloride and bicarbonate

126 transport. We submerged the tissue with $40 \mathrm{ml} \mathrm{HEPES}$ buffered saline with $10 \mu \mathrm{M}$ bumetanide

127 as previously described (3). $100 \mu \mathrm{M}$ methacholine was added to stimulate submucosal gland

128 secretion $(1,3)$.

130 Imaging interaction of S. aureus and mucus in pig tracheas. S. aureus strains were grown

131 overnight in tryptic soy broth (TSB) with $10 \mu \mathrm{g} / \mathrm{ml}$ Cam to maintain selection of GFP plasmids

132 (Table 1). We added $40 \mu \mathrm{L}$ of stationary phase GFP-S. aureus to the $40 \mathrm{ml}$ HEPES buffered

133 saline. In some experiments, we added 1:10,000 red fluorescent nanospheres (FluoSpheres,

134 Molecular Probes) to identify mucus strands $(1,3)$. We used a 25x (Nikon Apo LWD, Water, NA 
1.1) objective and a Nikon A1R confocal microscope. We quantitated the GFP signal on the epithelial surface and compared to the GFP signal on mucus strands. We determined the speed of individual S. aureus cells on these pig tracheas using Imaris software (Bitplane) and compared the speed of free bacteria to attached bacteria.

Quantifying the interaction of S. aureus and mucus by competition assay. Pig Gastric

141 Mucin (type III, sialic acid 0.5-1.5\%, Sigma \#M1778, Lot SLBL7748V) was dissolved in

142 phosphate buffered saline, $\mathrm{pH}$ 7. Mucin concentrations ranging from $4 \mu \mathrm{g} / \mathrm{mL}$ to $8 \mathrm{mg} / \mathrm{mL}$ were

143 produced by serial dilution. $100 \mu \mathrm{L}$ of diluted mucin was added to non-treated 96 -well black

144 optical plates (Thermo Fisher \#265301). Methylcellulose (MP Biomedicals \#155492, Lot 6257E),

145 heparin sulfate (Sigma \#H3149-250KU, Lot SLBS9674), and bovine submaxillary mucin (Sigma

146 \#M3895-1G, Lot SLBS0651V) were obtained commercially and prepared similarly as

147 antagonists of bacterial attachment. To dissolve methylcellulose, we autoclaved it as a dry

148 powder and dissolved it in PBS by stirring overnight at $4{ }^{\circ} \mathrm{C}$.

150 For in vitro binding assays, we diluted stationary-phase GFP S. aureus to OD of 0.6 in PBS.

151 Each strain of $S$. aureus had similar GFP signal per cell. $50 \mu \mathrm{L}$ of $S$. aureus were added per well 152 following dilution. Bacteria were allowed to attach for 1 hour at $37^{\circ} \mathrm{C}$. The mucin suspension 153 and any bacteria not attached to the plastic surface were removed by aspiration. We performed 154 two rounds of washing with PBS followed by suctioning. The residual fluorescence was assayed 155 using a SpectraMaxi3X plate reader with settings optimized for GFP.

157 Statistical analysis. For each experiment, we used 4 replicate wells for each condition.

158 Following experiments, we calculated the average GFP fluorescence from replicate wells and 159 plotted the average fluorescence versus $\log _{10}$ of the final concentration of binding antagonist 160 measured in $\mathrm{mg} / \mathrm{mL}$. We used GraphPad Prism version 7 to perform 4-parameter logistic 
161 regression, allowing for calculation of $\mathrm{IC}_{50}$. We rejected experiments from further analysis if

162 there were not at least 2 top and bottom values for fitting the logistic regression. For summary

163 statistics, we used $I_{50}$ values from independent experiments performed on different days. For

164 comparison of $\mathrm{IC}_{50}$ values, we used log-transformed data for statistical tests. For non-normally

165 distributed data sets, we used non-parametric statistical tests as appropriate.

167 Results

168 S. aureus attaches to mucus strands in pig tracheas.

169 In a previous study, we observed that mucus strands fail to detach after they emerge from

170 submucosal glands in CF pig tracheas (3). If bacteria attach to these mucus strands, they may

171 be retained in the airway. We obtained tracheas from newborn wild type pigs, submerged them

172 in bicarbonate-free HEPES buffered saline and treated with bumetanide. These conditions block

173 chloride and bicarbonate secretion, simulating CF airway conditions (3). We added

174 methacholine to stimulate mucus secretion by submucosal glands. To these preparations, we

175 added stationary phase $S$. aureus expressing GFP to test whether these bacteria associate with

176 mucus strands, or if they bind directly to the epithelium (Figure 1). We selected LAC, a

177 community-acquired strain of MRSA (CA-MRSA), as our model organism. CA-MRSA has

178 recently increased in prevalence within the CF population (17).

180 To help identify mucus strands, we added red fluorescent nanospheres as previously described

$181(1,3)$. In the presence or absence of nanospheres, GFP S. aureus was predominantly located

182 on mucus strands (Figure $1 \mathrm{~A}-\mathrm{C}$ ). Under these experimental conditions, mucus strands move

183 slowly and do not properly release from submucosal gland ducts (3). S. aureus attached to

184 these mucus strands had average speed less than $1 \mathrm{~mm} / \mathrm{min}$, whereas $S$. aureus that were not

185 attached to strands moved more quickly, indicating that few were attached to the surface

186 epithelium (Figure 1D, Supplemental Videos 1 and 2). 


\section{Quantifying bacteria-mucus interaction by competition assay.}

189 Mucus strands form a discontinuous mesh rather than a complete gel over the airway surface

$190(1,2)$. Such a discontinuous layer may not prevent bacteria from reaching the epithelial surface.

191 However, our confocal microscopy observations indicate that when S. aureus are added to pig

192 tracheas, they accumulate on these mucus strands rather than on the epithelial surface. This

193 might indicate that mucus strands function as competitors with the epithelial surface for bacterial

194 binding, similar to a decoy receptor. Once bacteria attach to mucus strands, ciliary beating

195 transports the aggregate of mucus and bacteria from the lungs. To quantitate the adherent

196 function of mucus, we designed a competition assay to measure how efficiently standardized

197 mucins prevent the attachment of bacteria to a standardized surface without the confounding

198 effect of ciliary flow.

200 We used microtiter plates as a standardized surface for attachment. GFP-labeled S. aureus

201 were added to these plates in the presence of variable concentrations of pig gastric mucin. We

202 incubated the suspension of bacteria and mucin for one hour at $37^{\circ} \mathrm{C}$, allowing bacteria to

203 associate with either the mucin or the plastic surface. Bacteria that did not attach to the plastic

204 surface, but instead associated with mucin in solution, were removed by aspiration and

205 washing. We quantitated residual bacteria attached to the surface with a fluorescent plate

206 reader (Figure 2).

208 We first compared the attachment of wild-type LAC and mutant strains to plastic in the absence

209 of mucin. We compared LAC to an isogenic mutant lacking Sortase A ( $\Delta s r t A)(12)$, which

210 catalyzes attachment of $S$. aureus surface proteins to the cell wall, and $\operatorname{Mgr} A(\Delta m g r A), a$

211 transcriptional regulator of adhesin expression (13). All three strains had similar attachment to

212 plastic prior to removal of unattached bacteria. $\Delta$ srtA had significantly decreased GFP signal 
213 following washing and aspiration, indicating defective attachment to plastic (Figure 3A). We did

214 not study it further. By contrast, the GFP signal from $\triangle m g r A$ was indistinguishable from LAC.

216 Mucin prevents bacterial attachment to plastic in a dose-dependent manner. When pig

217 gastric mucin was added to wells with bacteria, there was a saturating dose-dependent

218 reduction in attachment of wild-type LAC to plastic (Figure 3B). Using 4-parameter logistic

219 regression, we determined that the $\mathrm{IC}_{50}$ of pig gastric mucin as an inhibitor of wild type $S$.

220 aureus attachment to plastic was approximately $1.6 \times 10^{-2} \mathrm{mg} / \mathrm{mL}$.

221

222

MgrA is necessary for normal mucin interactions. Using this method, we compared LAC with $\Delta m g r A$, a strain with significantly altered surface adhesin expression (13). Although both LAC and $\triangle m g r A$ had similar binding capacity for the microtiter dish surface in the absence of mucin (Figure 3A), we observed differences in absorption to the plastic surface in the presence of mucin (Figure 3B). Significantly higher concentrations of pig gastric mucin were required to block attachment of $\Delta m g r A$, as visualized by the rightward shift in the inhibition curve. We repeated these studies using a partially complemented $\Delta m g r A$ strain (13) and found that partial restoration of MgrA partially normalized the interaction with mucin (Figure 3C).

validate the observed genotype-dependent binding differences were specific to mucin, we compared adhesion of LAC and $\triangle m g r A$ to mucin versus relevant biologic polymers:

234 methylcellulose, bovine submaxillary mucin, and heparin sulfate. Methylcellulose is used as a 235 mucin substitute in pharmacologic products such as artificial tears (18). We compared it to 236 mucin as both share viscoelastic physical properties and repeating saccharide units. Bovine 237 submaxillary mucin is a secreted mucin with a notably higher sialic acid content than porcine 238 gastric mucin (up to $30 \%$ vs. $1 \%$, respectively) $(19,20,21)$. We tested whether the increase in 
239 sialic acids displayed by BSM would lead to altered attachment by $\Delta m g r A$, since SraP was

240 previously shown to interact with sialic acid (22). Heparin sulfate is a linear polysaccharide

241 abundantly secreted at mucosal surfaces. We compared it to mucin as it shares similar

242 glycosylated residues but carries a stronger negative charge than mucin due to its sulfated

243 moieties.

245 Figure 4 summarizes our results. We found the altered binding phenotype observed in the

$246 \Delta m g r A$ mutant was specific to mucins. Both porcine gastric mucin and Bovine submaxillary

247 mucin exhibited statistically significant $I_{50}$ differences between $L A C(W T)$ and $\Delta m g r A$ strains.

248 Conversely, methylcellulose and heparin sulfate failed to recapitulate bacterial genotypic

249 differences in mucin adhesion.

251 These studies demonstrated that mucin is a poorer inhibitor of $\Delta m g r A$ attachment compared to

252 the wild type strain LAC. We considered three possible explanations for this phenomenon. 1)

253 MgrA is needed for the expression of classical S. aureus surface adhesins (13); 2) MgrA

254 suppresses expression of surface proteins that block interaction with mucin; or 3) MgrA induces

255 the expression of adhesins that directly bind mucin.

257 A S. aureus mutant lacking conventional adhesins has normal mucin interaction. Some of

258 the most well studied adhesins in S. aureus are ClfA, ClfB, and the fibronectin binding proteins

$259 \quad A$ and $B(F n b p A B)$. These adhesins have broad roles in binding fibrinogen, fibronectin, and

260 other extracellular matrix components, and have been linked to nasal colonization, clumping

261 and general S. aureus virulence properties (23). Using a mutant defective in all these adhesins

262 (LAC $\triangle c / f A B \triangle f b n A B)$, we tested interactions with mucin. We found that the inhibition curve of

263 pig gastric mucin versus this clumping factor mutant was indistinguishable from wild-type LAC 
264 (Figure 5A). Thus, the presence or absence of these important adhesins does not explain the

265 altered interaction of $\Delta m g r A$ with pig gastric mucin.

267 Deficiency in Ebh and SraP suppress $\Delta$ mgrA mucin interaction phenotype. MgrA functions

268 as a transcriptional repressor that blocks the expression of the large surface proteins Ebh and

269 SraP in the S. aureus LAC strain (13). We hypothesized that increased expression of one of

270 these surface proteins was responsible for the decreased mucin binding observed in $\Delta m g r A$.

271 Therefore, we tested the interaction of mucin with $\Delta m g r A$ lacking either Ebh, SraP, or both

272 (Figure $5 \mathrm{~B}$ and C). We found that deficiency in Ebh or SraP alone had minimal effect of mucin

273 interaction compared to the parental $\Delta m g r A$ strain. However, the loss of both Ebh and SraP

274 completely restored binding to the $\triangle m g r A$ strain. These data are most consistent with Ebh and

275 SraP functioning as interchangeable factors that inhibit bacterial interaction with mucin. This is

276 analogous to their function as anti-clumping factors (13). 


\section{Discussion}

279 S. aureus respiratory infections remain a major problem for patients with CF. It is well

280 documented that infection with methicillin-resistant strains of $S$. aureus is associated with

281 worsening CF disease progression $(6,7)$, and newer treatments for CF have had minimal effect

282 on the prevalence of $S$. aureus $(24,25)$. Moreover, $S$. aureus is a major cause of respiratory

283 disease in children and adults without CF $(26,27,28)$. Although $S$. aureus may colonize

284 asymptomatic individuals (29), S. aureus is a prevalent cause of community-acquired

285 pneumonia, especially following influenza infection (30-32) and has high case fatality rates (27,

$28633,34)$.

S. aureus binds airway mucus. Because of its importance as a respiratory pathogen, it is

289 crucial to understand how $S$. aureus initiates infections. We found that when introduced into

290 explanted airways, S. aureus accumulates on strands of mucus arising from submucosal

291 glands. When liquid secretion from these glands is blocked pharmacologically, the $S$. aureus

292 that attach to these strands may not be cleared efficiently (3). Previous studies of S. aureus

293 showed minimal contact with ciliated mucosal surface in human nasal biopsies (35) and that

294 attachment could be decreased with lectin or monosaccharide competition (36), consistent with

295 bacterial interaction with mucus.

297 Mucus is described as "sticky," but its adhesive properties are seldom quantified. The

298 development of a competition assay enabled us to measure the strength of the interaction

299 between mucus and bacteria. Using the $\mathrm{IC}_{50}$ of competition curves as an estimate of the affinity

300 of $S$. aureus for mucin, we found that the interaction with wild-type $S$. aureus is relatively weak

$301\left(\sim 10^{-2} \mathrm{mg} / \mathrm{mL}\right)$. However, mucin production is abundant and thus it may have high capacity for

302 removing inhaled bacteria. Since $S$. aureus can stimulate mucin synthesis $(37,38)$, initial 
303 infection could amplify the number of potential binding bacterial sites. This may perpetuate

304 infection in CF, where the release of mucus is impaired (3).

Bacterial surface factors regulate attachment and mucus interaction. How does $S$. aureus

307 adhere to respiratory mucus? Differences between bacterial strains isolated from patients

308 suggest genetic factors control the interaction of $S$. aureus with mucus (39). We predicted that

309 genes regulating the display of adhesins may alter the attachment properties of $S$. aureus (23).

310 Most of the classical surface adhesins are added to the $S$. aureus cell wall by sortase-

311 dependent transpeptidation (12). However, we observed a deficiency in attachment to our

312 standardized surface with $\triangle$ srtA S. aureus and did not study them further. MgrA regulates the

313 expression of some adhesins on the bacterial cell wall $(13,15)$. In the absence of MgrA, higher

314 concentrations of mucin were required to block attachment of $S$. aureus to microtiter plates,

315 consistent with decreased interaction of $S$. aureus with mucin. The decreased interaction of

$316 \Delta m g r A$ with mucin was not related to well-known adhesins ClfA, ClfB, or FnbpAB, as a different

317 strain lacking these surface proteins had normal interaction with mucin. Instead, the binding

318 phenotype of $\Delta m g r A$ depended on Ebh and SraP, which MgrA normally represses. We

319 speculate that these large surface proteins sterically block the interaction of glycans from mucin

320 side chains with low affinity receptors on the bacterial cell wall. This theory parallels recent work

321 demonstrating decreased adhesion of $\Delta m g r A$ S. aureus to various host molecules, including

322 fibrinogen, fibronectin, and collagen (15). This may also be a mechanism for the anti-clumping

323 activity of these large bacterial surface proteins (13).

325 S. aureus interaction with mucin may weaken with greater negative charge.

326 Previous studies of $S$. aureus showed its attachment to mucin was only weakly inhibited by N-

327 acetylneuraminic acid (11), and could be augmented by neuraminidase treatment (40). This

328 implies a low affinity state between $S$. aureus and sialic acid, likely explained by charge 
329 repulsion between teichoic acids on the bacterial cell wall and sialic acid residues on mucins.

330 We found that bovine submaxillary mucin, which has higher sialic acid content had decreased

331 interaction with $S$. aureus compared to pig gastric mucin. Heparin sulfate, a polyanionic polymer

332 had even weaker interaction with $S$. aureus. By contrast, there was stronger interaction of $S$.

333 aureus with methylcellulose, a neutral polysaccharide. Future studies using $S$. aureus strains

334 with mutations in teichoic acid biosynthesis may be useful for further testing the effect of charge

335 repulsion between mucins and the bacterial cell wall.

\section{Advantages}

338 Here, we report a quantitative, reproducible method for measuring the interaction of bacteria 339 with mucus. This method could be adapted to studying a larger number of mutants or clinical 340 isolates, and it could be adapted to study a variety of mucins or mucin analogs. Applying this

341 method, we can identify $S$. aureus mutants with defective attachment to a standard surface and 342 can quantitate the relative affinity of different $S$. aureus strains for mucin.

\section{Limitations}

345 The method we report does not capture the complexity of an in vivo or ex vivo airway 346 epithelium, in which antimicrobial proteins, innate immune cells, and an abundance of 347 macromolecules other than mucin may influence bacterial colonization. Furthermore, we did not 348 replicate the cilia-directed flow and continuous secretion of mucin seen in airway infections.

349 More specifically to mucin, pig gastric and bovine submaxillary mucins are standardized, 350 commercially available mucin glycoproteins, but they have been denatured and do not retain 351 their original viscoelastic properties. On the other hand, airway mucins are highly ordered $(1,2)$

352 and possess their native molecular properties. Our competition method for measuring

353 interaction of mucin and bacteria is indirect and requires intact attachment of bacteria to the 354 microtiter plate. However, because these mucins are easily washed off the plate surface, we 
355 found the indirect method was more feasible than direct interaction studies and did not require

356 additional steps such as chemical modification of the mucins to conjugate them to a surface.

358 Conclusions

359 Our study shows that $S$. aureus attaches to strands of mucus. These strands are poorly cleared

360 in CF. With a competition assay, we show that the affinity of $S$. aureus for mucin is relatively

361 weak $\left(10^{-2} \mathrm{mg} / \mathrm{mL}\right)$ and may be regulated by MgrA through repression of Ebh and SraP. This

362 method will enable further study of bacterial factors involved the interaction with host mucins.

363

\section{Acknowledgements}

365 This work was funded in part by NIH K08 HL136927 (AJF), CFF FISCHE16I0 (AJF), and NIH

366 Al083211 (ARH). CPP was supported a fellowship for medical students NIH T35 HL007485 40.

367 We thank Michael Welsh for his constructive feedback at several stages of this project.

368

369 Author Contributions

370 Conceived and designed studies: AJF, ARH

371 Performed experiments: CPP, CRS, PDA, NA, ALC

372 Wrote first draft of paper: CPP, AJF

373 Edited paper: CPP, AJF, ARH 
bioRxiv preprint doi: https://doi.org/10.1101/2020.04.09.035261; this version posted April 10, 2020. The copyright holder for this preprint (which was not certified by peer review) is the author/funder, who has granted bioRxiv a license to display the preprint in perpetuity. It is made available under aCC-BY-NC-ND 4.0 International license.

377 Table 1. Bacterial Strains and plasmids used in this study

\begin{tabular}{lll}
\hline Name & Genotype/Properties & Reference \\
\hline Strain & & \\
\hline LAC & Wild type, USA 300 CA MRSA, Erm ${ }^{\mathrm{S}}$ & Crosby (13) \\
\hline AH3455 & LAC $\Delta m g r A$ & Crosby (13) \\
\hline AH4480 & LAC $\Delta$ srtA & This work \\
\hline AH3485 & LAC $\Delta m g r A \varnothing 11::$ LL29erm mgrA & Crosby (13) \\
\hline AH4393 & LAC $\Delta$ clfA $\Delta f n b A B$ & Deng (41) \\
\hline AH4413 & LAC $\Delta$ clfA $\Delta$ fnbAB clfB::Tn & Deng (41) \\
\hline AH3798 & LAC $\Delta m r g A \Delta e b h$ sraP::Tn & Crosby (13) \\
\hline AH3481 & LAC $\Delta m g r A \Delta e b h$ & Crosby (13) \\
\hline AH3811 & LAC $\Delta m g r A ~ s r a P:: T n$ & Crosby (13) \\
\hline & &
\end{tabular}

Plasmid

pCM29 sGFP expression vector, Cam $^{R} \quad$ Pang (42) 

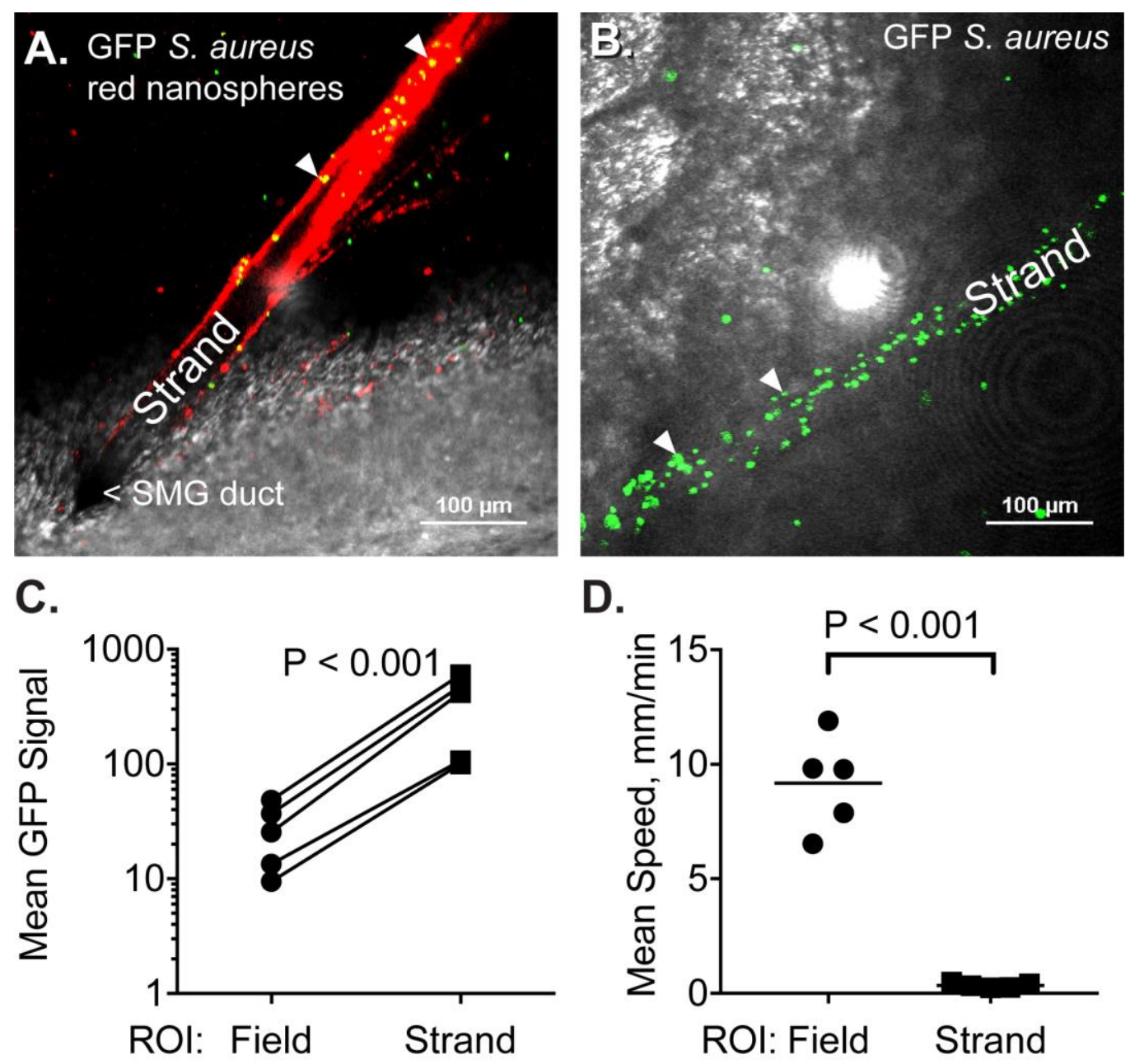

D.

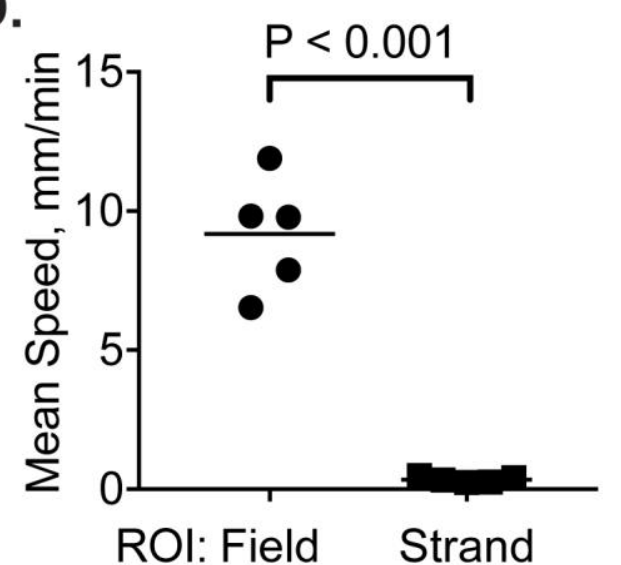

S. aureus

380 Figure 1. Attachment of GFP-S. aureus to mucus strands in pig tracheas. A. Confocal

381 micrograph shows a strand of mucus that is labeled with red fluorescent nanospheres. The

382 strand arises from a submucosal gland (SMG) duct. GFP-labeled S. aureus cells colocalize with

383 nanospheres and appear yellow. Arrowheads indicate two of the attached S. aureus cells. B.

384 Attachment of $S$. aureus to a mucus strand in the absence of nanospheres. C. Mean GFP

385 signal by region of interest (ROI), either within the mucus strand or elsewhere in the field. Lines

386 connect measurements made within the same experiment for 5 independent experiments. D.

387 Mean speed of individual S. aureus depending on whether bacteria are attached to the strand or 388 are elsewhere in the field. Each dot represents a unique experiment. 

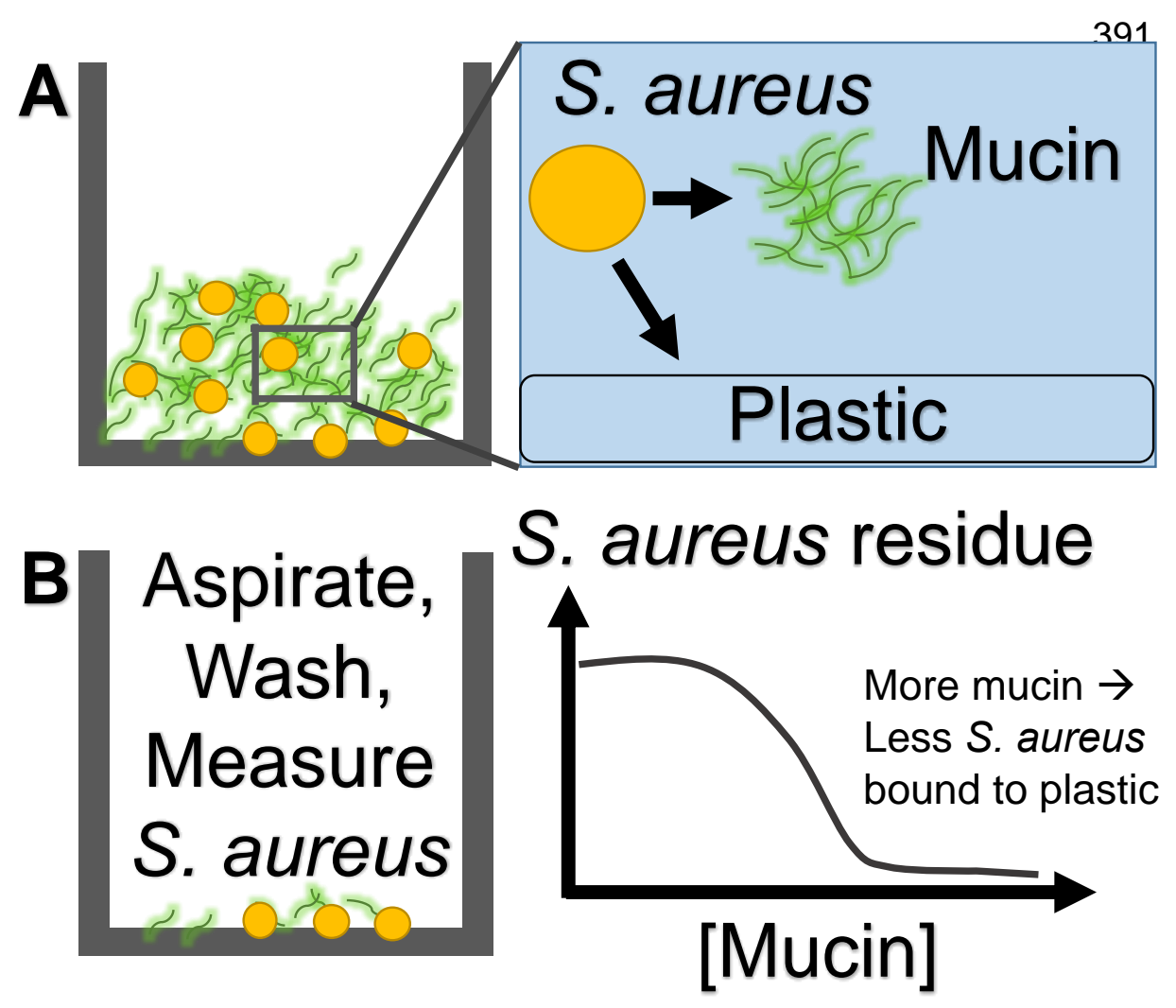

392 Figure 2. Schematic depiction of adherence assay. S. aureus is added to microtiter plate

393 wells containing varying concentrations of mucin and allowed to incubate for one hour. In this

394 scenario, the bacteria may either interact with the plastic surface or with the mucin, which

395 functions as a competitor. After an hour incubation, the bacteria-mucin suspensions are

396 aspirated, the microtiter plates are washed, and the remaining bacteria that are attached to the

397 plastic are measured by their GFP fluorescence. 
A

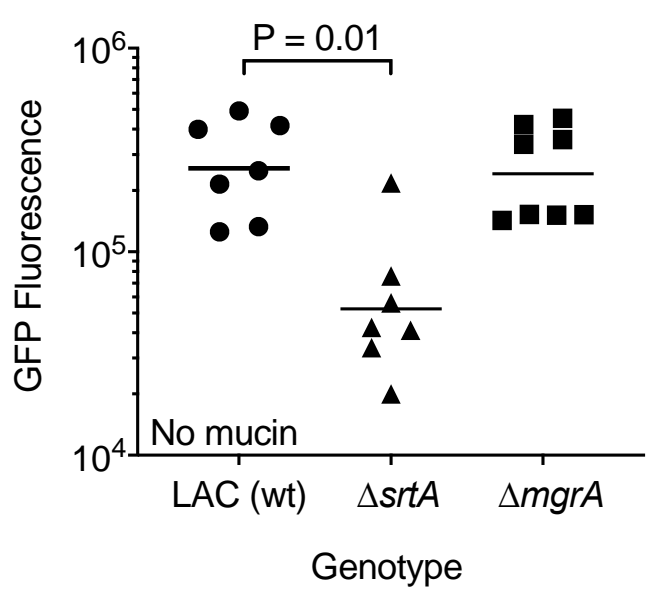

C



Genotype

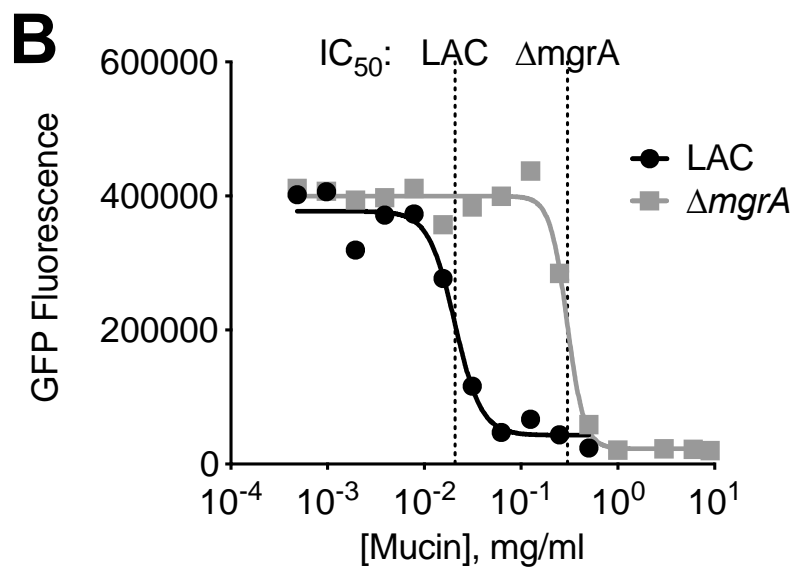

Figure 3. Detection of different attachment phenotypes by mucin competition assay. A.

Attachment of $S$. aureus strains to microtiter plates as measured by residual GFP fluorescence attachment was indistinguishable from isogenic LAC (wild type). Each point represents an independent experiment. $P$ values were calculated by Kruskal-Wallis test, comparing mutants 
409 Complementation of $m g r A$ partially restores mucin interaction. Data show $\mathrm{IC}_{50}$ of mucin versus

410 LAC, $\Delta m g r A$, and $\Delta m g r A$ with a wild-type copy of $m g r A$ integrated elsewhere on the

411 chromosome. The $\mathrm{IC}_{50}$ of mucin for attachment of $\Delta m g r A$ was significantly higher than both wild

412 type and the complemented strain. Complementation did not fully restore the wild-type

413 interaction with mucin. (1-way repeated measured ANOVA, $\mathrm{N}=8$ experiments, paired by date

414 of experiment).

415

416 


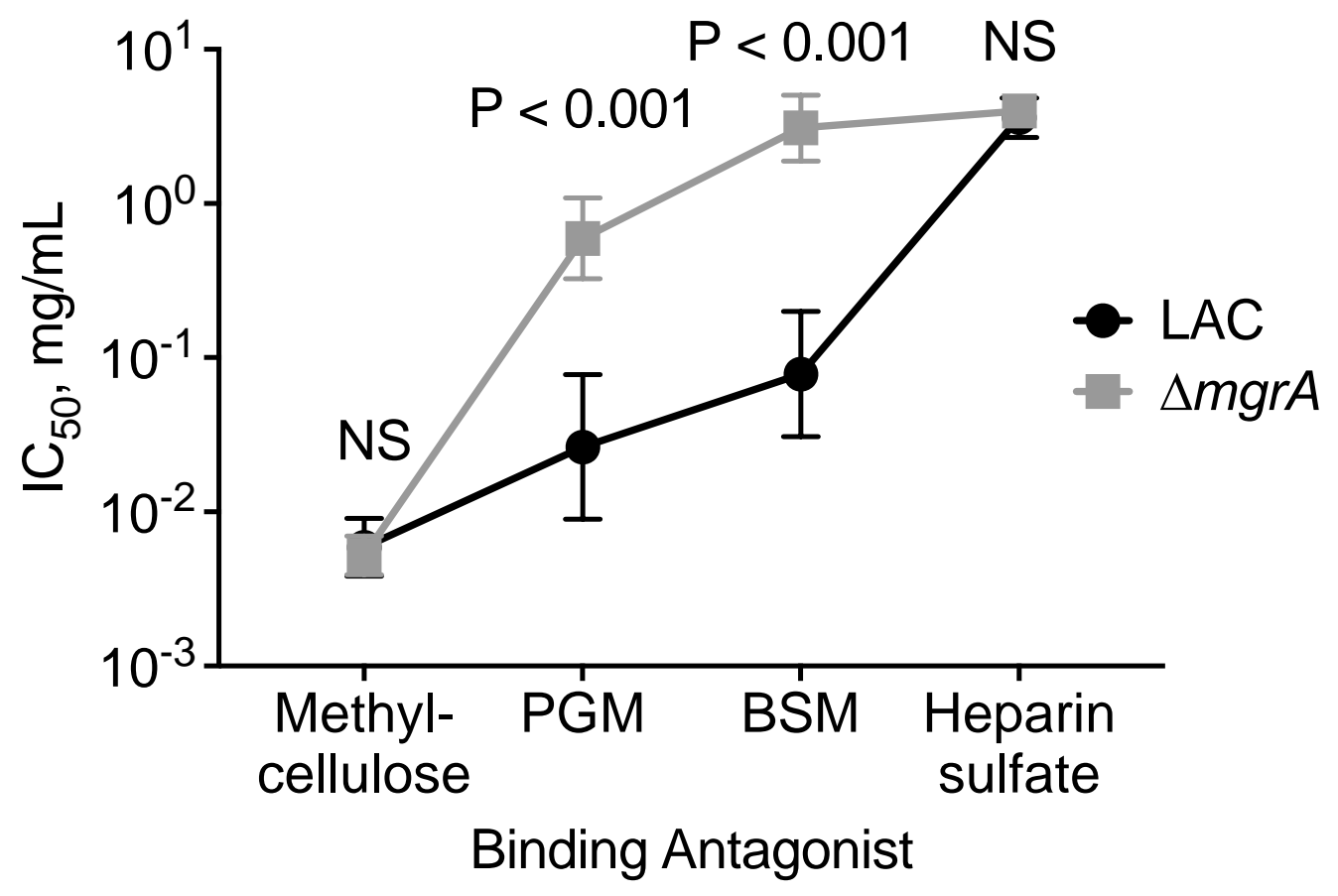

418 Figure 4. Differences between LAC and $\Delta m g r A$ are apparent in mucins, but not other

419 biological polymers. Methylcellulose, pig gastric mucin, bovine submaxillary mucin, and

420 heparin sulfate were tested for inhibitory activity against $S$. aureus attachment. $I C_{50}$ values for

421 each polymeric substance is shown for versus LAC (black circles) and $\Delta m g r A$ (gray squares).

422 Symbols represent mean and standard error, calculated on logarithmic transformed data.

423 Number of unique experiments per condition: 5 (Methylcellulose), 12 (PGM), 8 (BSM), and 3

424 (Heparin sulfate). Differences between LAC and $\Delta m g r A$ were observed with PGM and BSM. 

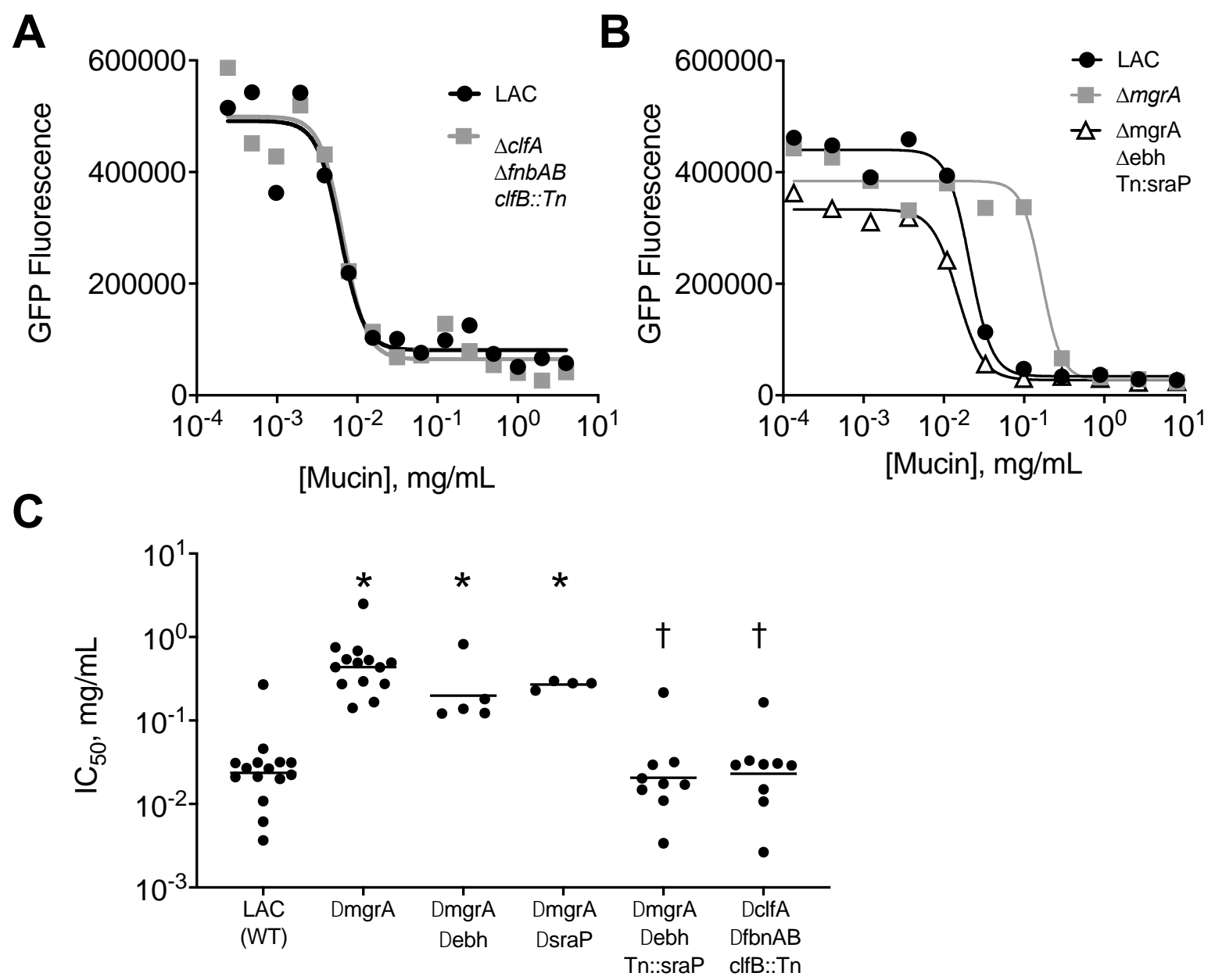

427 Figure 5. Regulation of S. aureus mucin interaction by adhesins downstream of MgrA.

428 A. Dose-dependent inhibition of S. aureus attachment in the presence and absence of four

429 classical adhesins. Representative data compare LAC (black) and an isogenic strain deficient in

$430 \quad c l f A, c l f B, f n b A$, and $f n b B$ (gray). There were no statistical differences between the two strains

431 under these conditions. B. Normalized interaction of $\Delta m g r A$ with pig gastric mucus in the

432 absence of Ebh and SraP. In this representative experiment, pig gastric mucin blocked

433 attachment of a triple mutant strain $\Delta m g r A \Delta e b h$ sraP::Tn (white triangles) at similar

434 concentration as was observed with the wild type LAC (black circles). Both strains had lower

$435 \quad \mathrm{IC}_{50}$ compared to $\triangle m g r A$ (gray squares). C. Summary data show $\mathrm{IC}_{50}$ of pig gastric mucin

436 versus a series of $S$. aureus mutants. Each dot is an independent experiment, bars represent 
bioRxiv preprint doi: https://doi.org/10.1101/2020.04.09.035261; this version posted April 10, 2020. The copyright holder for this preprint (which

was not certified by peer review) is the author/funder, who has granted bioRxiv a license to display the preprint in perpetuity. It is made available under aCC-BY-NC-ND 4.0 International license.

437 geometric mean. 1-way ANOVA with post-hoc comparisons to LAC or $\triangle m g r A$ controls. * $\mathrm{P}<$

$438 \quad 0.01$ vs LAC, † $\mathrm{P}<0.01$ vs $\Delta m g r A$.

439 
440 Supplemental Video 1. Confocal microscopy of excised pig trachea demonstrates reduced

441 speed of $S$. aureus attached to mucus. Pig tracheas were treated with bumetanide while

442 submerged in buffer lacking bicarbonate, slowing the emergence of mucus. Red fluorescent

443 nanospheres were added to identify demonstrate mucus strands, which emerge from

444 submucosal gland ducts. Green color indicates S. aureus that express green fluorescent

445 protein. Unattached S. aureus are transported by ciliary flow. Many S. aureus are attached to

446 the mucus strand. Their movement is slow compared to the unattached bacteria. Video is

447 shown in real time.

448 Supplemental Video 2. Confocal microscopy of excised pig trachea shows an attachment

449 event by S. aureus. Pig trachea was prepared similarly to the previous video, with red

450 fluorescent nanospheres used to illuminate mucus strands. GFP expressing $S$. aureus were

451 added. The video pauses at $10 \mathrm{~s}$, when a new green particle of $S$. aureus appears immediately

452 below the gland duct orifice. The video is slowed to $25 \%$ of normal speed until the $S$. aureus

453 appears on the mucus strand (20 s). The S. aureus subsequently remains on the mucus strand. 


\section{References}

456 1. Fischer AJ, Pino-Argumedo MI, Hilkin BM, Shanrock CR, Gansemer ND, Chaly

457 AL, Zarei K, Allen PD, Ostedgaard LS, Hoffman EA, Stoltz DA, Welsh MJ, Alaiwa MHA. 2019. Mucus strands from submucosal glands initiate mucociliary transport

2. Ostedgaard LS, Moninger TO, McMenimen JD, Sawin NM, Parker CP, Thornell morphologic structures in airways. Proc Natl Acad Sci U S A 114:6842-6847.

3. Hoegger MJ, Fischer AJ, McMenimen JD, Ostedgaard LS, Tucker AJ, Awadalla MA, Moninger TO, Michalski AS, Hoffman EA, Zabner J, Stoltz DA, Welsh MJ.

4. Salsgiver EL, Fink AK, Knapp EA, LiPuma JJ, Olivier KN, Marshall BC, Saiman Cystic Fibrosis. Chest 149:390-400.

471 5. Goss CH, Muhlebach MS. 2011. Review: Staphylococcus aureus and MRSA in cystic fibrosis. J Cyst Fibros 10:298-306.

473 6. Dasenbrook EC, Checkley W, Merlo CA, Konstan MW, Lechtzin N, Boyle MP. 2010. Association between respiratory tract methicillin-resistant Staphylococcus aureus and survival in cystic fibrosis. JAMA 303:2386-92.

476 7. Dasenbrook EC, Merlo CA, Diener-West M, Lechtzin N, Boyle MP. 2008. 
479 8. Muhlebach MS, Heltshe SL, Popowitch EB, Miller MB, Thompson V, Kloster M,

480

481

482

483

484

485

486

487

488

489

490

491

492

493

494

495

496

497

498

499

500

501

Ferkol T, Hoover WC, Schechter MS, Saiman L. 2015. Multicenter Observational

Study on Factors and Outcomes Associated with Various Methicillin-Resistant

Staphylococcus aureus Types in Children with Cystic Fibrosis. Ann Am Thorac

Soc 12:864-71.

9. Muhlebach MS, Beckett V, Popowitch E, Miller MB, Baines A, Mayer-Hamblett N, Zemanick ET, Hoover WC, VanDalfsen JM, Campbell P, Goss CH. 2016.

Microbiological efficacy of early MRSA treatment in cystic fibrosis in a randomised controlled trial. Thorax doi:10.1136/thoraxjnl-2016-208949.

10. Heo SM, Choi KS, Kazim LA, Reddy MS, Haase EM, Scannapieco FA, Ruhl S. 2013. Host defense proteins derived from human saliva bind to Staphylococcus aureus. Infect Immun 81:1364-73.

11. Sanford BA, Thomas VL, Ramsay MA. 1989. Binding of staphylococci to mucus in vivo and in vitro. Infect Immun 57:3735-42.

12. Mazmanian SK, Liu G, Ton-That H, Schneewind O. 1999. Staphylococcus aureus sortase, an enzyme that anchors surface proteins to the cell wall. Science 285:760-3.

13. Crosby HA, Schlievert PM, Merriman JA, King JM, Salgado-Pabon W, Horswill AR. 2016. The Staphylococcus aureus Global Regulator MgrA Modulates Clumping and Virulence by Controlling Surface Protein Expression. PLoS Pathog 12:e1005604.

14. Luong TT, Newell SW, Lee CY. 2003. Mgr, a novel global regulator in Staphylococcus aureus. J Bacteriol 185:3703-10. 
502 15. Kwiecinski JM, Crosby HA, Valotteau C, Hippensteel JA, Nayak MK, Chauhan AK, Schmidt EP, Dufrene YF, Horswill AR. 2019. Staphylococcus aureus adhesion in endovascular infections is controlled by the ArIRS-MgrA signaling

16. Novick RP. 1991. Genetic systems in staphylococci. Methods Enzymol 204:587636.

17. Akil N, Muhlebach MS. 2018. Biology and management of methicillin resistant Staphylococcus aureus in cystic fibrosis. Pediatr Pulmonol doi:10.1002/ppul.24139.

511 18. McCann LC, Tomlinson A, Pearce El, Papa V. 2012. Effectiveness of artificial tears in the management of evaporative dry eye. Cornea 31:1-5.

513 19. Snary D, Allen A. 1971. Studies on gastric mucoproteins. The isolation and characterization of the mucoprotein of the water-soluble mucus from pig cardiac

20. Straus DC, Unbehagen PJ, Purdy CW. 1993. Neuraminidase production by a Pasteurella haemolytica A1 strain associated with bovine pneumonia. Infect

519 21. Madsen JB, Sotres J, Pakkanen KI, Efler P, Svensson B, Abou Hachem M, Arnebrant T, Lee S. 2016. Structural and Mechanical Properties of Thin Films of Bovine Submaxillary Mucin versus Porcine Gastric Mucin on a Hydrophobic

523 22. Yang YH, Jiang YL, Zhang J, Wang L, Bai XH, Zhang SJ, Ren YM, Li N, Zhang 
Structural insights into SraP-mediated Staphylococcus aureus adhesion to host cells. PLoS Pathog 10:e1004169.

23. Foster TJ, Geoghegan JA, Ganesh VK, Hook M. 2014. Adhesion, invasion and evasion: the many functions of the surface proteins of Staphylococcus aureus. Nat Rev Microbiol 12:49-62.

530 24. Heltshe SL, Mayer-Hamblett N, Burns JL, Khan U, Baines A, Ramsey BW, Rowe SM. 2015. Pseudomonas aeruginosa in cystic fibrosis patients with G551DCFTR treated with ivacaftor. Clin Infect Dis 60:703-12.

25. Singh SB, McLearn-Montz AJ, Milavetz F, Gates LK, Fox C, Murry LT, Sabus A, Porterfield HS, Fischer AJ. 2019. Pathogen acquisition in patients with cystic

26. Wunderink RG, Waterer G. 2017. Advances in the causes and management of community acquired pneumonia in adults. BMJ 358:j2471.

27. Miles F, Voss L, Segedin E, Anderson BJ. 2005. Review of Staphylococcus fibrosis receiving ivacaftor or lumacaftor/ivacaftor. Pediatr Pulmonol

542 28. Geng W, Yang Y, Wu D, Zhang W, Wang C, Shang Y, Zheng Y, Deng L, Fu Z, Li X, Yu S, Shen X. 2010. Community-acquired, methicillin-resistant Staphylococcus aureus isolated from children with community-onset pneumonia 
2008. Detection of 11 common viral and bacterial pathogens causing communityacquired pneumonia or sepsis in asymptomatic patients by using a multiplex reverse transcription-PCR assay with manual (enzyme hybridization) or automated (electronic microarray) detection. J Clin Microbiol 46:3063-72.

30. Murray RJ, Robinson JO, White JN, Hughes F, Coombs GW, Pearson JC, Tan HL, Chidlow G, Williams S, Christiansen KJ, Smith DW. 2010. Communityacquired pneumonia due to pandemic $A(\mathrm{H} 1 \mathrm{~N} 1) 2009$ influenzavirus and methicillin resistant Staphylococcus aureus co-infection. PLoS One 5:e8705.

556 31. Rice TW, Rubinson L, Uyeki TM, Vaughn FL, John BB, Miller RR, 3rd, Higgs E, pandemic influenza A virus and bacterial coinfection in the United States. Crit

560 32. Rothberg MB, Haessler SD. 2010. Complications of seasonal and pandemic influenza. Crit Care Med 38:e91-7.

33. Maskell NA, Batt S, Hedley EL, Davies CW, Gillespie SH, Davies RJ. 2006. The bacteriology of pleural infection by genetic and standard methods and its mortality significance. Am J Respir Crit Care Med 174:817-23.

565 34. Schreiber MP, Chan CM, Shorr AF. 2011. Bacteremia in Staphylococcus aureus pneumonia: outcomes and epidemiology. J Crit Care 26:395-401.

567 35. Shuter J, Hatcher VB, Lowy FD. 1996. Staphylococcus aureus binding to human nasal mucin. Infect Immun 64:310-8. 
569 36. Sakarya S, Ertugrul MB, Ozturk T, Gokbulut C. 2010. Effect of pharynx epithelial cells surface desialylation on receptor-mediated adherence of Staphylococcus aureus. J Appl Microbiol 108:1313-22.

572 37. Lemjabbar H, Basbaum C. 2002. Platelet-activating factor receptor and ADAM10 mediate responses to Staphylococcus aureus in epithelial cells. Nat Med 8:41-6.

574 38. Kim YO, Jung MJ, Choi JK, Ahn do W, Song KS. 2011. Peptidoglycan from Staphylococcus aureus increases MUC5AC gene expression via RSK1-CREB pathway in human airway epithelial cells. Mol Cells 32:359-65.

577 39. Trivier D, Houdret N, Courcol RJ, Lamblin G, Roussel P, Davril M. 1997. The binding of surface proteins from Staphylococcus aureus to human bronchial mucins. Eur Respir J 10:804-10.

580 40. Ricciuto J, Heimer SR, Gilmore MS, Argueso P. 2008. Cell surface O-glycans 581 limit Staphylococcus aureus adherence to corneal epithelial cells. Infect Immun 76:5215-20.

583 41. Deng L, Schilcher K, Burcham LR, Kwiecinski JM, Johnson PM, Head SR, 584 Heinrichs DE, Horswill AR, Doran KS. 2019. Identification of Key Determinants of Staphylococcus aureus Vaginal Colonization. mBio 10.

586 42. Pang YY, Schwartz J, Thoendel M, Ackermann LW, Horswill AR, Nauseef WM. polymorphonuclear neutrophils. J Innate Immun 2:546-59. 